\title{
Meconium-stained liquor and its impact on maternal and neonatal outcome
}

\author{
Shweta Kashikar ${ }^{1}$, Monika K. Kotpalliwar ${ }^{2 *}$, Pooja R. Uttawar ${ }^{3}$
}

\author{
${ }^{1}$ Department of Obstetrics and Gynecology, Consultant, Mumbai, Maharashtra, India \\ ${ }^{2}$ Department of Obstetrics and Gynecology, Datta Meghe Institute of Medical Sciences, Sawangi (Meghe) Wardha, \\ Maharashtra, India \\ ${ }^{3}$ Department of Obstetrics and Gynecology, Consultant, Yavatmal, Maharashtra, India
}

Received: 30 January 2021

Accepted: 04 March 2021

\section{*Correspondence:}

Dr. Monika K. Kotpalliwar,

E-mail: monikakotpalliwar@gmail.com

Copyright: (C) the author(s), publisher and licensee Medip Academy. This is an open-access article distributed under the terms of the Creative Commons Attribution Non-Commercial License, which permits unrestricted non-commercial use, distribution, and reproduction in any medium, provided the original work is properly cited.

\section{ABSTRACT}

Background: Meconium stained amniotic fluid (MSAF) is predictor of adverse fetal outcomes. However, all fetuses with meconium passage do not have an adverse outcome and it is important to distinguish those who develop fetal distress promptly to prevent and intervene the sequalae accordingly. Hence this study was undertaken to study the grades of meconium stained liquor and its effect on intrapartum management and relation with maternal and neonatal outcome.

Methods: A prospective observational study was conducted on term pregnancy patients. Firstly, the detailed case history and examination was performed. When membranes ruptured the colour of liquor was noted. Fetal monitoring by cardiotocography (CTG) was done continuously throughout labour. The mode of delivery, cord $\mathrm{pH}$ and base excess were noted. After the delivery Apgar score and weight of the baby, need for resuscitation, neonatal intensive care unit (NICU) admissions and neonatal complications were noted.

Results: Of the 3117 deliveries conducted during the study period, 321 cases $(10.29 \%)$ had meconium stained liquor out of whom, 183 women (57\%) were with thin meconium and $138(43 \%)$ had thick meconium. There was no risk factor identified in the majority of women. The CTG was reactive in most women with thin meconium (71.5\%). There was a significantly higher incidence of pathological CTG's in women with thick meconium $(36.3 \%)(\mathrm{p}$ value = 0.001). There was no significant difference in birth weight Apgar scores, cord $\mathrm{pH}$, base excess in both the groups and rate of NICU admission.

Conclusions: Maximum patients with thin meconium and reactive CTG included in our study delivered normally with good neonatal outcome. However presence of thick meconium in addition to other factors such as fetal heart rate alterations should be viewed seriously as it may pose a potential danger for the baby.

Keywords: Fetal distress, Meconium stained amniotic fluid

\section{INTRODUCTION}

Meconium stained amniotic fluid (MSAF) is an alarming sign of fetal compromise and predictor of adverse fetal outcomes such as meconium aspiration syndrome and perinatal asphyxia, which leads to perinatal and neonatal morbidity and mortality. ${ }^{1-3}$ Meconium stained neonates are more prone to develop respiratory distress than neonates born with clear fluid. ${ }^{1}$ Meconium stained amniotic fluid significantly increase the rate of maternal complications such as meconium-laden amniotic fluid embolism, intrapartum chorioamnionitis, puerperal endometiritis, wound infection, increased risk of operative delivery and its complication. ${ }^{4,5}$ Incidence of MSAF ranges from $7-22 \%$ while meconium aspiration syndrome (MAS) occurs in approximately $5 \%$ of all cases 
of MSAF and more than $4 \%$ of MAS infants died accounting for $2 \%$ of all perinatal deaths. ${ }^{1,6,7}$

Meconium is a germ-free, thick, black-green, odourless material which is first recognized in the fetal intestine around 12 weeks of gestation and stores in the fetal colon throughout gestation. ${ }^{8}$ Passage of meconium in the newborn infants is a developmentally programmed incident; normally occurring within the first 24 to 48 hours after birth. However, the fetus may pass meconium in the amniotic fluid during pregnancy due to different reasons.

Passage of meconium is not common before 34 weeks of gestation, beyond that period the incidence gradually increases. $^{9}$ The exact etiology of meconium stained amniotic fluid is not clear. ${ }^{2}$ However MASF is more frequently seen in post term pregnancy or in growth restricted fetuses. ${ }^{7}$ Meconium passage could follow vagal stimulation from common but transient umbilical cord entrapment and resultant increased peristalsis and relaxation of anal sphincter. ${ }^{1,10}$ An increased incidence of meconium passage into the amniotic cavity is also noted in the presence of feto-maternal stress factors such as hypoxia and infection, independent of fetal maturation. Factors such as placental insufficiency, maternal hypertension, pre-eclampsia, oligohydramnios or maternal drug abuse (tobacco or cocaine) also result in, in-utero passage of meconium. ${ }^{7}$

Meconium stained liquor (MSL) is the passage of meconium by a fetus in utero during the antenatal period or in labour and is classified as significant MSL and nonsignificant MSL. Non-significant MSL is defined as a thin yellow or greenish tinged fluid; containing nonparticulate meconium whereas significant MSL is explained as dark green or black amniotic fluid that is thick and tenacious and consists lumps of meconium. ${ }^{2}$

The perinatal morbidity and mortality related to MSAF can be decreased if major risk factors are recognized early and closely monitoring of the labour and careful decisions are made about the timing and mode of delivery. ${ }^{2}$ Various measures have been done to prevent these complications like amnioinfusion, oropharyngeal suctioning, endotracheal intubation after birth etc. ${ }^{10}$

Since all fetuses with meconium passage in labour do not have an adverse outcome, it is important to distinguish those who develop fetal distress promptly and intervene accordingly to prevent meconium aspiration syndrome and sequelae. Meconium aspiration syndrome occurs when meconium-stained amniotic fluid is aspirated into the lungs of an infant prior to, during or immediately after birth. Meconium aspiration syndrome occurs in approximately $5 \%$ of infants born through meconiumstained amniotic fluid. ${ }^{11}$

Hence the present study of 'meconium-stained liquor and its impact on maternal and neonatal outcome' has been undertaken to study the grades of meconium stained liquor and its effect on intrapartum management and relation with maternal and neonatal outcome.

\section{METHODS}

The present study was a prospective observational study conducted from $1^{\text {st }}$ October 2010 to $30^{\text {th }}$ September 2012 after approval by the Ethics Committee Jehangir hospital.

\section{Inclusion criteria}

The inclusion criteria was live singleton pregnancy, term pregnancy (>37 completed weeks) and cephalic presentation.

\section{Exclusion criteria}

Exclusion criteria were pre-term pregnancy $(<37$ completed weeks), antepartum hemorrhage, abnormal presentation, previous caesarean section/myomectomy, multiple pregnancy, pregnancy with congenital malformations, intrauterine death.

All women admitted to Jehangir Hospital, who fulfilled the inclusion criteria and had meconium stained liquor during their labour were enrolled in the study after obtaining a written informed consent.

A detailed history regarding age, gravidity and parity, past obstetrical history, menstrual history, history of present pregnancy, history of medical and surgical disorders was noted. This was followed by a general and systemic examination and then an obstetrical examination was performed. The height of fundus, amount of liquor, presentation and fetal heart rate were noted. Along with this pelvic assessment was also performed.

Both women who came in spontaneous labour and in whom labour was induced were included. Labour induction was done with PGE1, PGE2 or oxytocin. When membranes ruptured spontaneously or when artificial rupture of membranes (ARM) was performed, the colour of liquor was noted. Women who developed meconium staining of liquor, during the course of labour were also included. Meconium was graded into thin and thick based on Patil et al criteria. ${ }^{12}$

Uterine contractions were assessed based on frequency, duration and intensity. Intrapartum continuous fetal heart monitoring was done using cardiotocography (CTG) tracing. Four parameters of CTG were taken into account i.e. rate, variability, deceleration and acceleration. Baseline fetal heart rate $<110 \mathrm{bpm}$ was considered as fetal bradycardia and $>160 \mathrm{bpm}$ was considered fetal tachycardia. A variability of $<5$ was taken as poor variability or loss of variability. Early, late and variable decelerations were noted. CTG was considered normal when all 4 parameters (rate, variability, deceleration, acceleration) were reassuring, suspicious when 1 
parameter was non reassuring and pathological when 2 or more than 2 parameters were non reassuring or one parameter was abnormal. Fetal monitoring by CTG was done continuously throughout labour. If the trace deteriorated the patient was reclassified and managed accordingly. The mode of delivery, cord $\mathrm{pH}$ and base excess were noted. After the delivery the condition of the baby was assessed using Apgar score and weight of the baby. Need for resuscitation, neonatal intensive care unit (NICU) admissions and neonatal complications were noted.

\section{Statistical method}

After collecting the data it was transferred into MS Excel (MS Office version 2007 developed by Microsoft, Redmond, WA) and then descriptive analysis and Chisquare test was used to determine the association and level of significance was kept below 0.05 .

\section{RESULTS}

Of the 3117 deliveries conducted during the study period, 321 cases $(10.29 \%)$ had meconium stained liquor (Table $1)$.

Table 1: Prevalence of meconium.

\begin{tabular}{|l|l|}
\hline Variable & N (\%) \\
\hline No. of deliveries during study period & $3117(100)$ \\
\hline No. of cases with MSL & $321(10.29)$ \\
\hline No. of cases with clear liquor & $2796(89.71)$ \\
\hline
\end{tabular}

Table 2: Meconium distribution $(n=321 ; 100 \%)$ in various parameters.

\begin{tabular}{|c|c|c|}
\hline Parameters & & $\mathbf{N}(\%)$ \\
\hline \multirow{4}{*}{ Age (in years) } & $<25$ years & $35(10.9)$ \\
\hline & $25-29$ years & $160(49.8)$ \\
\hline & $30-34$ years & $104(32.5)$ \\
\hline & $>35$ years & $22(6.8)$ \\
\hline \multirow{2}{*}{ Gravidity } & Primigravida & $193(60.1)$ \\
\hline & Multigravida & $128(39.9)$ \\
\hline \multirow{2}{*}{ Gestational age } & $37-40$ weeks & $128(67.9)$ \\
\hline & $\geq 40$ weeks & $103(32.1)$ \\
\hline \multirow{6}{*}{ Risk factors } & Post-datism & $103(32)$ \\
\hline & IUGR & $32(9.9)$ \\
\hline & Obstetric cholestasis & $10(3.1)$ \\
\hline & Pre-eclampsia & $18(5.6)$ \\
\hline & Oligohydramnios & $14(4.6)$ \\
\hline & No risk factors & $144(4.8)$ \\
\hline \multirow{2}{*}{ Onset of labour } & Spontaneous & $206(64.2)$ \\
\hline & Induced & $115(35.8)$ \\
\hline
\end{tabular}

Majority of women belonged to the age group of 2529years. Majority of women $(67.9 \%)$ were between 37 40 weeks of gestation. There was no risk factor identified in the majority of women. Post-dated pregnancy was the commonest risk factor noted in the others. Most of the women had come in spontaneous labour. Only 35.8\% of women needed induction of labour (Table 2).

Out of 321 cases of meconium stained liquor, there were 183 women (57\%) with thin meconium and 138 women (43\%) with thick meconium (Table 3).

Table 3: Grades of meconium.

\begin{tabular}{|ll|}
\hline Meconium & Number of cases; $\mathbf{n}(\%)$ \\
\hline Thin & $183(57)$ \\
\hline Thick & $138(43)$ \\
\hline Total & $321(100)$ \\
\hline
\end{tabular}

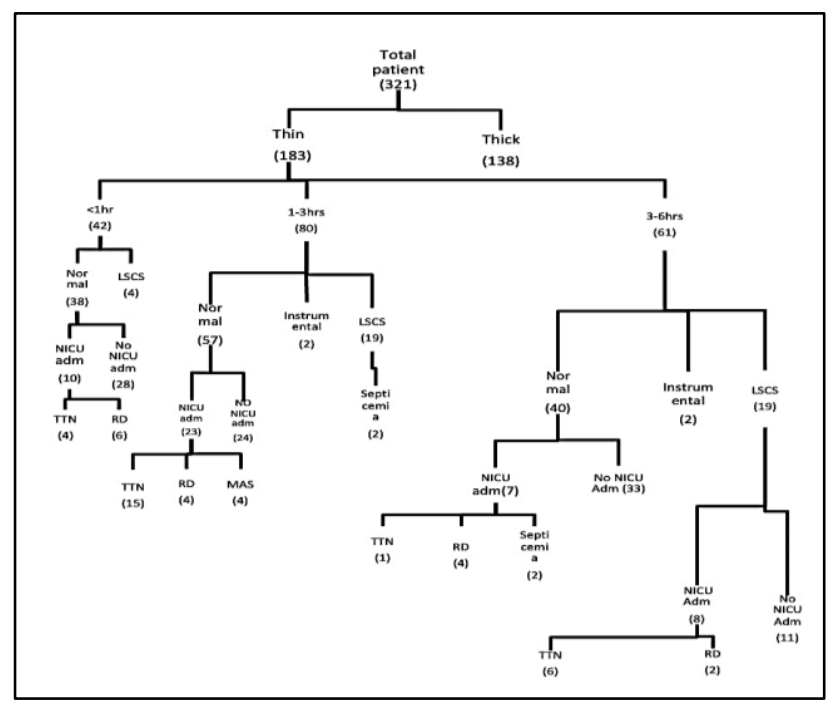

Figure 1: Summary flowchart of patients during study.

Meconium was diagnosed in most women in both groups of thin and thick meconium at $3-5 \mathrm{~cm}$ dilatation of cervix. ( $n=103 ; 56.3 \%$ and $n=63 ; 45.7 \%$, respectively). The CTG was reactive in most women with thin meconium $(71.5 \%)$. There was a significantly higher incidence of pathological CTG's ( $p$ value $=0.001$ ) in women with thick meconium $(36.3 \%)$ compared to women with thin meconium $(0.1 \%)$. In thick meconium $50 \%$ cases and $23 \%$ with thin were delivered within one hour. Majority of patients with thin meconium had normal delivery. Rate of caesarean section was $40 \%$ higher in thick meconium and this was statistically significant $(\mathrm{p}=0.002 *)$. Rates of instrumental delivery were $2.2 \%$ in thin meconium. Birth weight across both the groups was similar ( $p$ value $=0.961)$.

Similarly, there was no significant difference in the Apgar scores, cord $\mathrm{pH}$ and base excess in both the groups. Grade of meconium did not affect the rate of NICU admission. The most common complication seen in both the groups was transient tachypnoea of the newborn (TTN). The incidence of meconium aspiration syndrome in thin meconium was $2.2 \%$ and in thick 
meconium was $5.8 \%$. This difference did not achieve statistical significance. In thin meconium, $160(87.4 \%)$ babies had cord $\mathrm{pH}>7.2$ and $23(12.6 \%)$ babies had acidosis at birth. Out of those, 3 babies were admitted in NICU for transient tachypnoea of newborn. In thick meconium, $121(87.7 \%)$ babies had cord $\mathrm{pH}>7.2$ and 17 $(12.3 \%)$ babies had acidosis at birth. Out of those, 9 babies were admitted in NICU, 5 for transient tachypnoea of newborn, 2 for MAS and 2 for septicaemia (Table 4 and Figure 1).

Table 4: Comparison of grades of meconium in various parameters.

\begin{tabular}{|c|c|c|c|c|}
\hline \multirow{2}{*}{ Parameters } & & \multicolumn{2}{|c|}{ Grades of meconium } & \multirow{2}{*}{$P$ value } \\
\hline & & Thin & Thick & \\
\hline \multirow{3}{*}{ Cervical dilatation } & $<3 \mathrm{~cm}$ & $25(13.7 \%)$ & $41(29.7 \%)$ & $0.04 *$ \\
\hline & $3-5 \mathrm{~cm}$ & $103(56.3 \%)$ & $63(45.7 \%)$ & 0.062 \\
\hline & $6 \mathrm{~cm}$ & $55(30 \%)$ & $34(24.6 \%)$ & 0.074 \\
\hline \multirow{3}{*}{ Cardiotocography (CTG) } & Normal & $131(71.5 \%)$ & $47(34 \%)$ & $0.001 *$ \\
\hline & Suspicious & $51(27.9 \%)$ & $41(29.7 \%)$ & 0.297 \\
\hline & Pathological & $1(0.1 \%)$ & $50(36.3 \%)$ & $0.001 *$ \\
\hline \multirow{3}{*}{$\begin{array}{l}\text { Interval between detection } \\
\text { of meconium and delivery }\end{array}$} & $<1$ hour & $42(23 \%)$ & $69(50 \%)$ & $0.024 *$ \\
\hline & 1-3 hours & $80(43.7 \%)$ & $55(39.9 \%)$ & 0.093 \\
\hline & 3.1-6 hours & $61(33.3 \%)$ & $14(10.1 \%)$ & $0.037 *$ \\
\hline \multirow{3}{*}{ Mode of delivery } & Normal & $139(75.9 \%)$ & $55(39.9 \%)$ & $0.029 *$ \\
\hline & Caesarean & $40(21.9 \%)$ & $83(60.1 \%)$ & $0.002 *$ \\
\hline & Instrumental & $4(2.2 \%)$ & $0 \%$ & NA \\
\hline \multirow{4}{*}{ Birth weight } & $<2500 \mathrm{gm}$ & $20(11 \%)$ & $12(8.6 \%)$ & 0.488 \\
\hline & $2501-3000 \mathrm{gm}$ & $54(30 \%)$ & $37(26.8 \%)$ & 0.554 \\
\hline & $3001-3500 \mathrm{gm}$ & $63(35 \%)$ & $67(48.7 \%)$ & $0.013 *$ \\
\hline & $3501-4000 \mathrm{gm}$ & $44(24 \%)$ & $22(15.9 \%)$ & 0.068 \\
\hline \multirow{2}{*}{ Apgar score } & $<7$ at 1 minute & $25(13.6 \%)$ & $21(15.2 \%)$ & 0.571 \\
\hline & $<7$ at 5 minute & $0 \%$ & $1(0.7 \%)$ & NA \\
\hline \multirow{2}{*}{ Cord pH } & $<7.2$ & $23(12.6 \%)$ & $17(12.3 \%)$ & 0.924 \\
\hline & $>7.2$ & $160(87.4 \%)$ & $121(87.7 \%)$ & 0.947 \\
\hline \multirow{2}{*}{ Base excess } & $<-8$ & $158(86.3 \%)$ & $118(85.5 \%)$ & 0.825 \\
\hline & -8 to -12 & $25(13.7 \%)$ & $20(14.5 \%)$ & 0.831 \\
\hline \multirow{2}{*}{ NICU admission } & Required & $50(27.3 \%)$ & $49(35.5 \%)$ & 0.081 \\
\hline & Not required & $133(72.7 \%)$ & $89(64.5 \%)$ & 0.076 \\
\hline \multirow{6}{*}{ Neonatal complications } & TTN & $16(8.7 \%)$ & $15(10.9 \%)$ & 0.857 \\
\hline & Respiratory distress & $26(14.2 \%)$ & $19(13.8 \%)$ & 0.297 \\
\hline & MAS & $4(2.2 \%)$ & $8(5.8 \%)$ & 0.248 \\
\hline & Septicemia & $4(2.2 \%)$ & $6(4.3 \%)$ & 0.527 \\
\hline & PPHN & $0 \%$ & $1(0.7 \%)$ & NA \\
\hline & Mortality & $0 \%$ & $0 \%$ & NA \\
\hline
\end{tabular}

\section{DISCUSSION}

Meconium stained amniotic fluid has traditionally been considered as a marker of fetal distress or hypoxia. Its presence has very often led to interventions such as instrumental delivery or caesarean section. However, current literature suggests that the presence of meconium in amniotic fluid without fetal heart variation or fetal acidosis is not a sure sign of fetal distress and need not be an indication for active intervention. ${ }^{13}$

Of the 3117 deliveries conducted in the hospital in the study period, 321 cases fulfilling the inclusion criteria were included for the study.
The prevalence of MSAF in the present study was $10.29 \%$. A similar incidence of $8.6 \%, 10.2 \%$ and $10.1 \%$ respectively was noted by Ziadeh et al, Arulkumaran et al, and Hiremath et al in their studies respectively. ${ }^{14-16}$ Bhatia et al and Leonard et al noted a much lower incidence of MSAF. ${ }^{17,18}$

In the present study, amongst all the MSAF cases the incidence of thin meconium stained amniotic fluid was higher than thick which was similar to the findings of Chhabra et al. ${ }^{19}$

In our study $60.1 \%$ were primigravida and $39.9 \%$ were multigravida. According to Rao et al there was a decrease 
in incidence of meconium stained liquor as parity increased. ${ }^{20}$ However Gupta et al opined that parity did not influence the incidence of meconium stained amniotic fluid. ${ }^{21}$

Various risk factors have been associated with a higher incidence of meconium stained amniotic fluid. In our study, most women did not have an identifiable risk factor. However, some of the risk factors noted were post-datism, obstetric cholestasis, oligohydramnios, IUGR and pre-eclampsia. $32 \%$ of women in our study had postdated pregnancies. This can be explained by the fact that hormone motilin secretion increases as gestational age advances. Increase in the level of motilin stimulates intestinal peristalsis which leads to passage of meconium. ${ }^{22}$ The findings of current study were in accord with Bhatia et al and Hiremath et al. ${ }^{16,17}$ However Shaikh et al and Sasikala et al noted a much lower incidence of $11.3 \%$ and $16 \%$ respectively of postdate deliveries in meconium stained amniotic fluid. 23,24

In present study $9.9 \%$ of patients with meconium stained amniotic fluid had intrauterine growth restriction. Some studies like those by Hiremath et al and Sankhyan et al have reported a similar incidence of $13.2 \%$ and $15 \% .^{16,25}$ Others by Shaikh et al and Narli et al found a higher incidence of IUGR with meconium i.e. $16.5 \%$ to $20 \% .^{23,26}$

Obstetric cholestasis was found in $3.1 \%$ of women with meconium stained amniotic fluid. Whereas Pusl et al and Dang et al had an incidence of $27 \%$ and $40.4 \%$ respectively of meconium in their studies on intrahepatic cholestasis. $^{27,28}$

In pre-eclampsia there is vasoconstriction leading to placental insufficiency which in turn causes fetal distress. This could explain the association of pre-eclampsia and meconium stained fluid. In the present study, the incidence of pre-eclampsia was $5.6 \%$ which was line with Klufio et al however slightly higher incidence was reported by Khatun et al and Narli. ${ }^{3,26,29}$

The incidence of oligohydramnios in MSL was observed $4.6 \%$ in this study. Similarly, Bhatia et al in their study reported a $4 \%$ incidence of oligohydramnios. ${ }^{17}$ Blackwell et al however, stated that the frequency of meconium stained amniotic fluid at birth in term pregnancies was not related to amniotic fluid volume. ${ }^{30}$

During the study period, majority of women came in spontaneous labour. These findings are supported by many previous researches. ${ }^{29,31}$ More females with thin meconium was observed which is parallel to Ziadeh et al. ${ }^{14}$ Contrast to this, some studies have reported a higher incidence of thick meconium. ${ }^{21,32}$

In current study found that the dilatation of cervix at enrolment influenced the further course of labour and its management. Those with a higher dilatation had a lower caesarean section rate and those with a lesser dilatation had a higher caesarean section rate.

It was observed that maximum fetal heart rate abnormality (suspicious and pathological) was seen with thick meconium as compared with thin meconium stained amniotic fluid which was in line with findings of Ziadeh et al. ${ }^{14}$ CTG abnormalities at detection of meconium or developing later influenced the trial of labour and mode of delivery. Higher rates of suspicious or pathological CTG's precipitated action which is evidenced by a higher Caesarean section rate in the thick meconium group.

The mode of delivery was influenced by detection of meconium, dilatation of cervix, CTG abnormalities and gravidity of the patient. The incidence of Caesarean section was $40 \%$ higher in thick meconium and this was statistically significant. Literature reveals a large variation in caesarean section rates across various studies. Bhatia et al has reported similar rates. ${ }^{17}$ Shaikh et al on the other hand has reported a very high caesarean section rate of $82 \% .^{23}$ On the contrary a very low caesarean section rate has been observed by Ziadeh et al (14\%) in thick meconium. ${ }^{14}$

This study found that primigravidas had more caesareans and vaginal deliveries were more in multigravida. Similar findings were reported by Hiremath et al in their study. ${ }^{16}$

In our study, most of the babies had a birth weight between 2501-3500 gm. Some studies have reported a similar incidence of birth weight 2501-3500 gm. ${ }^{16,19}$ Fujikura observed an increased incidence of meconium staining with birth weight more than $3 \mathrm{~kg} .{ }^{33}$

The character of meconium did not influence the Apgar score at 1 minute or 5 minute in our study. Starks et al also made the same observation. ${ }^{32}$ Klufio et al in their study observed a low Apgar score $(<7)$ at 1 minute and at 5 minute in meconium group. ${ }^{29}$ Whereas Gupta et al reported that Apgar score $(<3)$ at 1 minute was significantly greater $(27 \%)$ in those with thick meconium. $^{21}$ Similarly, Khatun et al and Steer et al observed a significantly greater incidence of Apgar score below 7 at 1 minute and 5 minute in meconium stained amniotic fluid compared to clear amniotic fluid. ${ }^{3,34}$

Cord arterial $\mathrm{pH}$ has historically been used as a primary marker of hypoxic/ischaemic injury. Now-a-days base excess has more significant usefulness as it doesn't change significantly with respiratory acidosis and gestational age. ${ }^{35}$ Cord $\mathrm{pH}$ was measured at birth to document evidence of acidosis in neonates. In our study, $13.7 \%$ babies in the thin meconium group and $14.5 \%$ in the thick meconium group had a base excess between -8 and -12 . The mean base excess was -6.79 . Narli et al observed mean base excess in thin meconium as $-6.2 \pm 4.6$ and in thick meconium as $-11.6 \pm 7.4 .^{26}$ Jazayeri et al reported mean base excess in meconium as $-4.6 \pm 0.16 .^{36}$ Ziadeh et al observed a higher incidence of acidosis with 
thick meconium $(21 \%)$ as compared with thin meconium $(12 \%) .{ }^{14}$

During the study period, only 50 cases $(27.3 \%)$ in thin meconium and $49(35.5 \%)$ in thick meconium required NICU admission. Arulkumaran et al noted $4.2 \%$ with thin meconium and $11.7 \%$ NICU admission with thick meconium. ${ }^{15}$ Ziadeh SM noted $4 \%$ NICU admission with thin meconium and $13 \%$ with thick meconium. ${ }^{14}$ Klufio CA noticed $13.4 \%$ of babies with meconium were admitted to NICU. ${ }^{29}$

Other studies had a much higher rate of NICU admission. Hiremath PB found $80 \%$ babies from their study admitted to NICU and Bhatia et al noted $74.4 \%$ of NICU admission. ${ }^{16,17}$

In the present the study, $68.8 \%$ neonates were asymptomatic and only $31.6 \%$ of babies developed neonatal complications.

The most common complication seen in both the groups was transient tachypnoea of the new born which was $14.2 \%$ in thin and $13.8 \%$ in thick meconium group. The incidence of meconium aspiration syndrome in thin meconium was $2.2 \%$ and in thick meconium $5.8 \%$. This difference did not achieve statistical significance. There was no neonatal mortality in our study.

$26(14.2 \%)$ babies with thin meconium and $19(13.8 \%)$ babies with thick meconium developed transient tachypnoea of newborn. Chest sounds were clear. X ray chest showed prominent pulmonary vascular markings, over aeration and flat diaphragms. All these babies were admitted in the NICU and given supportive treatment including supplemental oxygen. Out of 45 babies, 7 babies required nasal oxygen therapy and remaining 38 babies were admitted only for observation. The condition resolved in 24-48 hours. Sasikala et al in their study observed that around $40 \%$ babies with meconium stained amniotic fluid had TTN. ${ }^{24}$

$16(8.7 \%)$ babies in thin meconium and $15(10.9 \%)$ babies in thick meconium group had mild respiratory distress where $\mathrm{x}$ ray chest was normal. Careful and frequent monitoring of heart and respiratory rate, oxygen saturation, $\mathrm{PaO}_{2}, \mathrm{PaCO}_{2}, \mathrm{pH}$, serum bicarbonate, electrolytes, glucose, haematocrit, blood pressure and temperature was done. Out of 31 babies with respiratory distress, 22 babies required nasal oxygen therapy and the remaining 9 babies settled with observation in 1-2 days and did not require ventilator support. Similarly, Bhat et al reported a $13.4 \%$ incidence of respiratory distress and Ziadeh et al $12 \% .{ }^{14,37}$ On the contrary, Sasikala et al observed a low incidence of $5.3 \%$ of respiratory distress in 150 cases of thick meconium stained amniotic fluid. ${ }^{24}$

$8(5.8 \%)$ babies with thick meconium and $4(2.2 \%)$ babies in thin meconium developed MAS. Treatment included supportive care with treatment of hypoxia and administration of antibiotics as per requirement. Oxygen saturation, $\mathrm{PaO}_{2}, \mathrm{PaCO}_{2}, \mathrm{pH}$, serum bicarbonate, electrolyte, glucose and haematocrit were measured. Blood pressure and temperature were monitored. All babies of meconium aspiration syndrome required mechanical ventilation. Their radiograms showed changes such as diffuse, asymmetric patchy infiltrations, area of consolidation and pneumonic patches. Most studies have reported a much higher incidence of MAS. Swain et al reported an incidence of $8.57 \%$, Patil et al gave an incidence of $12.9 \%$ and Bhatia et al observed MAS in $28.4 \%$. $^{12,16,17}$

Septicaemia was developed in around $3.1 \%$ babies who were given antimicrobial therapy for suspected or known pathogen with supportive care. Adequate oxygenation of tissue was maintained. 7 babies were treated with i.v. antibiotics. NICU stay was for 3-4 days. Similarly, Sasikala et al reported $3.8 \%$ incidence of septicaemia. ${ }^{24}$ Whereas Bhatia et al noted a very high incidence of septicaemia of $16 \% .^{17}$

Only $1(0.7 \%)$ case with thick meconium developed persistent pulmonary hypertension (PPHN). Initial management included oxygen administration and correction of acidosis, hypotension and hypercapnia. This baby required intubation and mechanical ventilation. Gradually the condition of the baby improved, ventilatory support was weaned off followed by oxygen support for 4 days. The baby recovered fully and was discharged from hospital on Day 6 of life. Narli et al noted a very high incidence of PPHN $10.5 \%$ in thick meconium. ${ }^{26}$

There was no neonatal mortality observed in present study. Neonatal mortality ranging from $0.5 \%$ to $1.7 \%$ have been reported by previous studies. ${ }^{14,37}$

Meconium in the amniotic fluid is an obstetric hazard which significantly increases risks of adverse neonatal outcomes. Women with meconium in the presence of normal fetal heart rate can be safely managed conservatively and allowed to deliver normally. Continuous fetal heart rate monitoring during labour can reduce adverse neonatal outcome.

\section{CONCLUSION}

The commonest risk factor associated with MSAF is postdatism. Other risk factors associated with MSAF are pre-eclampsia, IUGR and obstetric cholestasis. MSAF alone is not an indicator of fetal distress, especially in thin meconium stained amniotic fluid. Grading of meconium and availability of continuous intrapartum fetal heart rate monitoring play an important role in the management of patients with meconium stained amniotic fluid. Apart from this, outcome of labour depends upon gravidity, dilatation of cervix at detection of meconium and the intensity of labour pains. 
Many centres in our country lack facilities for electronic fetal heart rate monitoring and fetal scalp blood studies. In such conditions vigilant monitoring of patients is mandatory to reduce the incidence of caesarean section and morbidity associated with it in a low resource country like India.

If conditions are favourable, trial of labour can be given to most patients with meconium stained amniotic fluid with minimum neonatal complications. Maximum patients with thin meconium and reactive CTG included in our study delivered normally and had a good neonatal outcome. However presence of thick meconium in addition to other factors such as fetal heart rate alterations should be viewed seriously as it may pose a potential danger for the baby.

\section{ACKNOWLEDGMENTS}

We are thankful to the Department of Pediatrics, Jahangir Hopsital, Pune, Maharashtra, India and the patients who consented to participate in this study.

Funding: No funding sources

Conflict of interest: None declared

Ethical approval: The study was approved by the Institutional Ethics Committee

\section{REFERENCES}

1. Mohammad N, Jamal T, Sohaila A, Ali SR. Meconium stained liquor and its neonatal outcome. Pak J Med Sci. 2018;34(6):1392-6.

2. Addisu D, Asres A, Gedefaw G, Asmer S. Prevalence of meconium stained amniotic fluid and its associated factors among women who gave birth at term in Felege Hiwot comprehensive specialized referral hospital, North West Ethiopia: a facility based cross-sectional study. BMC Pregnanc Childbirth. 2018;18(1):429.

3. Khatun MHA, Arzu J, Haque E, Kamal M, Al Mamun MA, Khan MFH, et al. Fetal outcome in deliveries with meconium stained liquor. Bangladesh J Child Health. 2009;33(2):41-5.

4. Desai D, Maitra N, Patel P. Fetal heart rate patterns in patients with thick meconium staining of amniotic fluid and its association with perinatal outcome. Int J Reprod Contracept Obstet Gynecol. 2017;6(3):10305.

5. Sori D, Belete A, Wolde M. Meconium stained amniotic fluid: factors affecting maternal and perinatal outcomes at Jimma University specialized teaching hospital, south West Ethiopia. Gynecol Obstet. 2016;6(394):1-6.

6. Jain PG, Sharma R, Bhargava M. Perinatal outcome of meconium stained liquor in pre-term, term and post-term pregnancy. Indian J Obstet Gynecol Res. 2017;4(2):146-50.

7. Desai D, Chauhan K, Chaudhary S. A study of meconium stained amniotic fluid, its significance and early maternal and neonatal outcome. Int $\mathbf{J}$ Reprod Contracept Obstet Gynecol. 2013;2(2):190-3.

8. Parvin I, Khanam N, Alam A. Management practices in cases with meconium stained amniotic fluid (MSAF) Babies. 2008;6(12):102-5.

9. Mundhra R, Agarwal M. Fetal outcome in meconium stained deliveries. J Clin Diagn Res. 2013;7(12):2874-6.

10. Jain PG, Sharma R, Bhargava M. Perinatal outcome of meconium stained liquor in pre-term, term and post-term pregnancy. Indian J Obstet Gynecol Res. 2017;4(2):146-50

11. Walsh MC, Fanaroff JM. Meconium stained fluid: Approach to the mother and the baby. Clin Perinatol. 2007;34(4):653-65.

12. Patil KP, Swamy MK, Samatha K. A one year cross sectional study of management practice of meconium stained amniotic fluid and perinatal outcome. J Obstet Gynecol India. 2006;56:128-30.

13. Miller FC, Sacks DA, Yeh SY, Paul RH, Schifrin BS, Martin CB, et al. Significance of meconium during labor. Am J Obstet Gynecol. 1975;122:57380 .

14. Ziadeh SM, Sunna E. Obstetric and perinatal outcome of pregnancies with term labour and meconium stained amniotic fluid. Arch Gynecol Obstet. 2000;264:84-7.

15. Arulkumaran S, Yeoh SC, Gibb DMF, Ingemarsson I, Ratnam SS. Obstetric outcome of meconium stained liquor in labour. Singapor Med J. 1985;26(7):523-6.

16. Hiremath PB, Gane B, Meenal C, Bansal N, Ragaramya. The management practices and outcome of meconium stained amniotic fluid. Int $\mathrm{J}$ Biol Med Res. 2012;3(3):2204-7.

17. Bhatia P, Ela N. Fetal and neonatal outcome of babies in meconium stained amniotic fluid and meconium aspiration syndrome. J Obstet Gynecol India. 2007;57(7):501-4.

18. Leonard JL. The significance of meconium stained amniotic fluid in cephalic presentation. Obstet Gynecol. 1961;20(3):320-3.

19. Chhabra S, Dargan R, Nasare M. Post date pregnancies: management options. J Obstet Gynecol India. 2007;57(4):307-10.

20. Rao B, Chandrashekhar GS, Rao D, Hegde P, Ghate SV. Meconium stained amniotic fluid- a prospective study. Karnataka Paediatr J. 2011;25(1):21-6.

21. Gupta V, Bhatia BD, Mishra OP. Meconium stained amniotic fluid: Antenatal, intrapartum and neonatal attributes. J Indian Pediatr. 1996;33:293-7.

22. Benirschke K, Kaufmann P. Pathology of the Human Placenta. 4th edn. New York: Springer-Verlag; 2000:653-659.

23. Shaikh EM, Mehmood S, Shaikh MA. Neonatal outcome in meconium stained amniotic fluid- one year experience. J Pak Med Assoc. 2010;60(9):7114.

24. Sasikala A, Raghavan S, Mishra N, Khatoon S, Bupathy A, Rani R. Perinatal outcome in relation to 
mode of delivery in meconium stained amniotic fluid. Indian J Pediatr. 1995;62(1):63-7.

25. Sankhyan N, Sharma VK, Sarin R, Pathania K. Predictor of meconium stained amniotic fluid: a possible strategy to reduce neonatal morbidity and mortality. J Obstet Gynecol India. 2006;56(6):514-7.

26. Narli N, Kirimi E, Satar M. Evaluation and Management of Neonates with meconium stained amniotic fluid. East J Med. 2001;6(1):18-21.

27. Pusl T, Beuers U. Intrahepatic cholestasis of pregnancy. Orphanet J Rare Dis. 2007:1-6.

28. Dang A, Agarwal N, Bathla S, Sharma N and Balani S. Prevalence of liver disease in pregnancy and its outcome with emphasis on obstetrics cholestasis: an Indian scenario. J Obstet Gynecol India. 2010;60:413-8.

29. Klufio CA, Amoa AB, Kariwiga G, Rageau O. A case control study of meconium staining of amniotic fluid in labour at Port Moresby General Hospital to determine associated risk factors and perinatal outcome. Papua New Guinea Med J. 1996;39:297309.

30. Blackwell SC, Wolfe HM, Redman ME, Hassan SS, Berry SM, Treadwell MC, et al. Relationship between meconium staining and amniotic fluid volume in term pregnancies. Fetal Diagn Ther 2002;17(2):78-82.

31. Rathor AM, Singh R, Ramji S, Tripathi R. Randomized trial of amnioinfusion during labour with meconium stained amniotic fluid. $\mathrm{Br} \mathrm{J}$ Obstet Gynaecol. 2002;109(1):17-20.
32. Starks GC. Correlation of meconium stained amniotic fluid, early intrapartum fetal $\mathrm{pH}$ and Apgar score as predictors of perinatal outcome. Obstet Gynecol. 1980;56:604-9.

33. Fujikura T, Klionsky B. The significance of meconium staining. Am J Obstet Gynecol. 1975;121:45-50.

34. Steer PJ, Eigbe F, Lissauer TJ, Beard RW. Interrelationships among abnormal cardiotocograms in labors, meconium staining of the amniotic fluid, arterial cord blood $\mathrm{pH}$, and Apgar scores. Obstet Gynecol. 1989;74(5):715-21.

35. Shakira P. Umbilical cord article blood base excess as gold standard for foetal well-being screening test validity at term delivery. J Pak Med Assoc. 2010;60:347-50.

36. Jazayeri A, Politz L, Tsibris JC, Queen RN, Spellacy WN. Fetal erythropoietin levels in pregnancies complicated by meconium passage: does meconium suggest fetal hypoxia? Am J Obstet Gynecol. 2000;183(1):188-90.

37. Bhat RY, Rao A. Meconium stained amniotic fluid and meconium aspiration syndrome: a prospective study. Ann Trop Pediatr. 2008;28(3):199-203.

Cite this article as: Kashikar S, Kotpalliwar MK, Uttawar PR. Meconium-stained liquor and its impact on maternal and neonatal outcome. Int J Reprod Contracept Obstet Gynecol 2021;10:1629-36. 